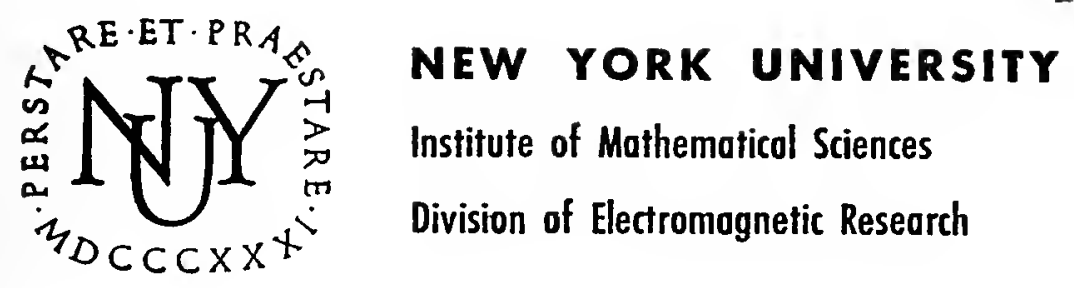

RESEARCH REPORT NO. EM-89

\title{
Asymptotic Formulas for Diffraction by Parabolic Surfaces
}

HARRY HOCHSTADT 



\title{
NEW YORK UNIVERSITY
}

Institute of Mathematical Sciences

Division of Electromagnetic Research

Research Report No. EM-89

ASYMPTOTIC FORMULAS FOR DIFFRACTION BY PARABOLIC SURF ACES

\author{
Harry Hochstadt
}
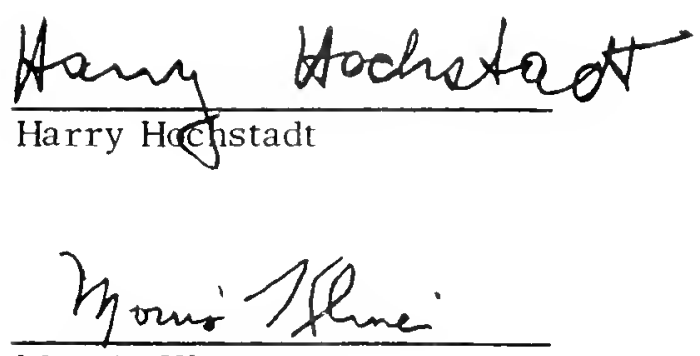

Morris Kline

Project Director

March, 1956

The research reported in this document has been made possible through

the support and sponsorship extended by the Air Forre Cambridge Re-

search Center, under Contract No. AF 19(122)-42. It is published

for technical information only and does not necessarily represent re-

commendations or conclusions of the sponsoring agency. 

1. Introduction

2. The caustic surface

3. The Green's function in parabolic coordinates

4. The Green's function in parabolic cylinder coordinates

5. Applications of the asymptotic formulas to diffraction problems

References
1

3

5

11

19

31

Abstract

A technique is discussed by which asymptotic solutions of reflection problems in paraboloidal and parabolic cylinder coordinates can be found for large wave numbers. This technique applies to reflections from the interior of parabolic and paraboloidal reflectors.

Detailed discussions are then given for the cases of reflection of incoming plane waves, waves emitted by sources at the focus, and waves emitted by sources on the axis of the reflector, for both coordinate systems. For the last problem the nature of the caustic surface and the behavior of the reflected wave on the caustic, off the caustic, and at the cusp of the caustic are discussed. 



\section{Introduction}

The purpose of this report is to discuss the asymptotic solutions of some diffraction problems in parabolic and paraboloidal coordinate systems, in the sense that the wave number $k$ will be large.

Buchholz $[1]$ has derived Groen's function representations in these coordinate systems. Using his formulas it is possible to derive the exact. solution to the problem of the reflection of a spherical wave on the inside of a paraboloidal mirror. This leads to a contour integral, which does not lend itself readily to asymptotic expansions. In the neighborhood of the cusp of the caustic surface the behavior of the reflected wave is such that the solution depends very strongly on how the cusp is approached. The exact solution indicates only one of several possible patterns of behavior.

Various techniques have been developed to find the asymptotic solutions of reflection problems. Keller, Lewis and Seckler ${ }^{[2]}$ have solved numerous problems, but not where caustic surfaces were involved. An approach to the asymptotic solution of diffraction problems in two dimensions where caustic surfaces are involved was discussed by Kay and Keller [3].

The technique developed in the present report allows one to construct asymptotic solutions of the wave equation

$$
\Delta u+k^{2} u=0
$$

where the boundary ralues, $\bar{u}$, are given on the inside surface of a paraboloid of revolution or a parabolic cylinder. The solution can be expressed by Green's theorem by the integral

$$
u=\iint \bar{u} \frac{\partial G}{\partial \bar{N}} d s .
$$


Asynptotic expansions of $\frac{\partial G}{\partial N}$ are used in the integrand to construct the solution. The resulting integrals can be evaluated readily when points of stationary phase can be found.

A similar technique is developed for problems where $\frac{\partial u}{\partial N}$ is prescribed on the boundary.

It should be observed that these asymptotic forms show that Huygens' principle has some solidity in these cases.

The following problems are treated:

a) reflection of incoming plane waves from the interior of $=$ paraboloid of revolution and a parabolic cylinder;

b) reflection of spherical waves due to a point source at the focus of a paraboloid of revolution, and reflection of cylindrical waves due to a line source at the focal line of a parabolic cylinder;

c) problems similar to b) except that the sources are on the axis of the reflector away from the focus or focal line.

In the last case different asymptotic expressions are obtained for points on and off the caustic and at the cusp of the caustic. 


\section{The canstic surface}

If one considers the case of a point source placed on the axis of the reflector one finds that the envelope of the reflected rays plays an important role. That envelope is called the caustic surface.

We will now derive the equation of the caustic surface and derive some of 1ts properties. Consider a $(\rho, z)$ coordinate system with a parabolic reflector given by the equation

$$
\rho^{2}=4 \eta(z+\eta)
$$

where $\eta$ denotes the focal length and the focus is placed at the origin. A point source is now placed at the point $(0, a)$ and it can be shown by geometrical optics that the ray which intersects the reflector at $(\bar{p}, \bar{z})$ will be reflected into the ourface and will intersect the axis at $(0, q)$, where

$$
q=\frac{(\bar{z}+2 \eta)^{2}}{a}
$$

The slope of the reflected ray is given by

$$
p=\frac{q-\bar{z}}{-\bar{p}}
$$

Therefore the equation of the reflected ray is given by

$$
z=q+p p
$$

This line must be tangent to the caustic surface at some point $\left(\rho_{c}, z_{c}\right)$, so that

$$
\begin{aligned}
& z_{c}-q+\rho_{c} p \\
& f^{\prime}\left(\rho_{c}\right)=p_{z}
\end{aligned}
$$

where $z=f(p)$ represents the caustic surface. Since one can asscciate one point $(\bar{\rho}, \bar{z})$ with every $\left(\rho_{c}, z_{c}\right)$ we can write 


$$
f^{\prime}\left(\rho_{c}\right)=\frac{d q}{d \bar{z}} \frac{d \bar{z}}{d \rho}+\rho_{c} \frac{d \rho}{d \bar{z}} \frac{d \bar{z}}{d \rho}+p=p
$$

from which we see that

$$
\begin{aligned}
& \rho_{c}=-\frac{q^{\prime}}{p^{\prime}}, \\
& z_{c}=\frac{p^{\prime} q-q^{\prime} p}{p^{\prime}},
\end{aligned}
$$

and since $q$ and $p$ are functions of $\bar{z}$ these represent the parametric equations of the caustic surface.

Those points where $\bar{z} \approx-\eta$ correspond to rays which are reflected from points near the vertex of the parabola. One can now show that for

$$
\begin{aligned}
& \bar{z} \approx-\eta, \\
& \bar{\rho} \approx 0
\end{aligned}
$$

the cauctic surface is given by

$$
\begin{aligned}
& \rho=\frac{\bar{\rho}^{-3}}{\eta(a+\eta)} \\
& z=\frac{\eta^{2}+\bar{\rho}^{2}}{a} \\
& \bar{\rho}^{2}=4 \eta(\bar{z}+\eta) .
\end{aligned}
$$

Now $\bar{\rho}$ can be eliminated and we obtain

$$
\left(z-\frac{\eta^{2}}{a}\right)^{3}=\frac{\eta^{2}}{a}\left(1+\frac{\eta}{a}\right)^{2} \rho^{2},
$$

which shows that at the point $\left(0, \frac{\eta^{2}}{a}\right)$ the caustic has a cusp. Figure 1 shows the general nature of the caustic surface.

We now consider the function

$$
R+r \text {, }
$$

where 


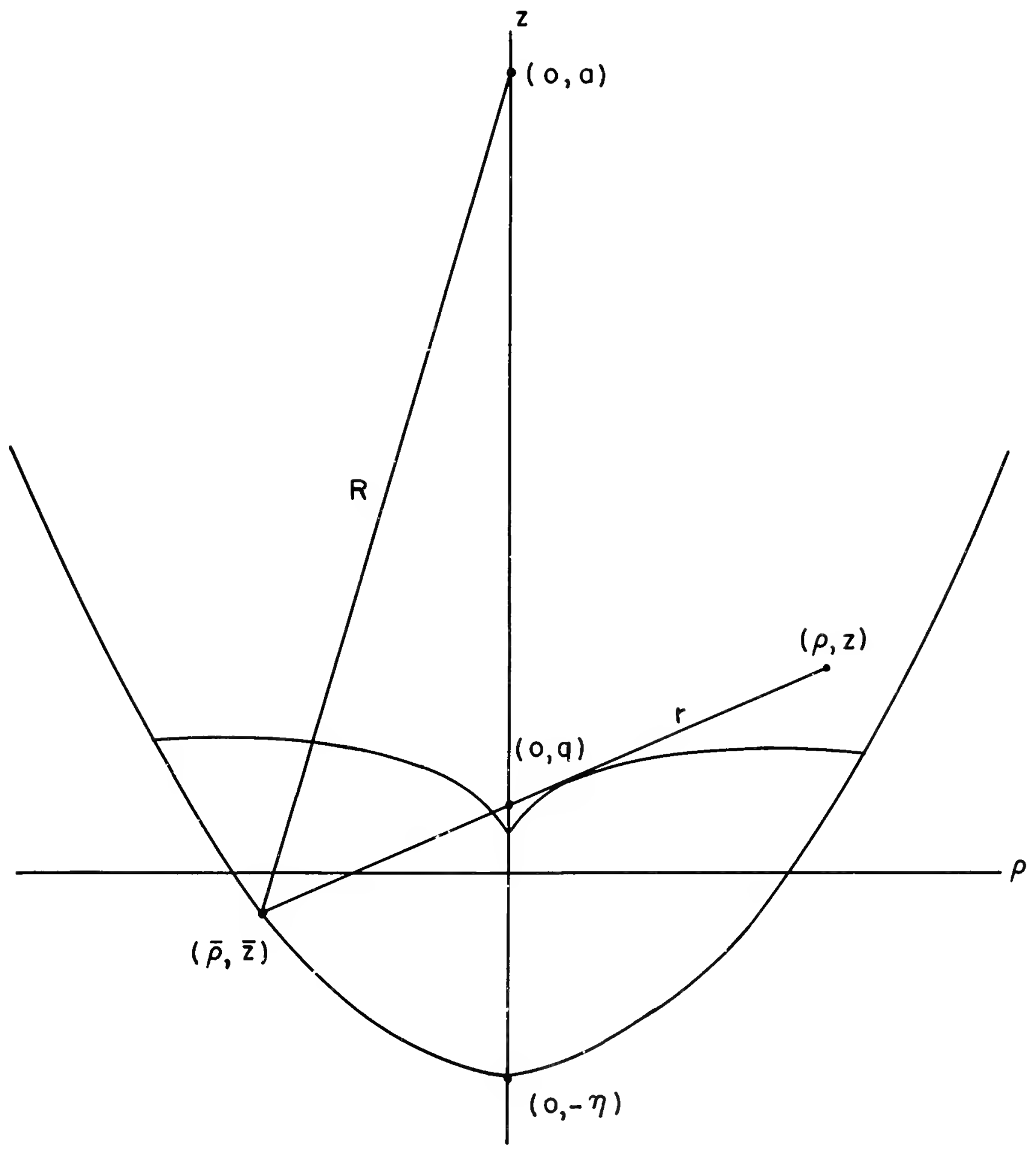

Fioure 1 


$$
\begin{aligned}
& R=\sqrt{(\bar{z}-a)^{2}+\bar{\rho}^{2}} \\
& r=\sqrt{(\bar{z}-z)^{2}+(\bar{\rho}-\rho)^{2}},
\end{aligned}
$$

and seek those values of $\bar{z}$ for which it is stationary. We will assume that $\bar{\rho}$ is negative, $(\rho, z)$ lies abcve the caustic, and $\rho$ is positive. Then $R+r$ must have three stationary points. Two of these can be located by geometrical optics, because they correspond to paths of least time. If it is possible to draw tangents from $(\rho, z)$ to the caustic, then the intersections of the tangents with the parabolic surface correspond to paths of least time. But since $\frac{d(R+r)}{d \bar{\rho}}$ is negative at $\bar{z}=-\eta, \bar{p}=0$ it follows that $R+r$ must have a local maximum for some point where $\vec{p}$ is less than $O$ but greater than the point corresponding to the path of least time. If $\rho=0$ then the local maximus corresponds to the point $(0,-\eta)$. In this case the stationary points are symmetrically located on the parabola.

As the point $(\rho, z)$ approaches the caustic, the two stationary points for $\bar{\rho}<0$ approach each other. Then in the limit they merge, so that not only $d(R+r) / d \bar{p}$ but also $d^{2}(R+r) / d \rho^{-2}$ vanishes. The stationary point is a point of inflection, so that $d^{3}(R+r) / d \rho^{-3}$ does not vanish.

For points below the caustic only one stationary point is left for which $\bar{p}>0$. For points on the axs and below the caustic that point is $(0,-\eta)$.

3. The Green's function in parabolic coordinates

The parabolic coordinate system can be defined by the transformation

$$
\begin{aligned}
& x=2 \sqrt{\xi \eta} \cos \phi \\
& y=2 \sqrt{\xi \eta} \sin \phi \\
& z=\xi-\eta .
\end{aligned}
$$

The surface $\eta=\eta_{a}$ defines a paraboloid whose axds coincides with the zaxis, and which includes the entire positive part of the 2 -axis. Similarly $\xi-\xi_{a}$ is also parabolic, but opens in the opposite direction. When expressed in parabolic coordinates, the wave quation $\Delta u+k^{2} u=0$ becomos 


$$
\frac{1}{2(\xi+\eta)}\left\{\frac{\partial}{\partial \xi} 2 \frac{\partial u}{\partial \xi}+\frac{\partial}{\partial \eta} 2 \frac{\partial u}{\partial \eta}+\frac{\xi+\eta}{\xi \eta} \frac{\partial^{2} u}{\partial \phi^{2}}\right\}+k^{2} u=0 .
$$

Separation of variables then leads to the system of ordinary differential equations

$$
\begin{aligned}
& \frac{d}{d \xi} \xi \frac{d f_{1}(\xi)}{d \xi}+\left(k^{2} \xi-\frac{\mu^{2}}{4 \xi}-21 k \chi\right) f_{1}(\xi)=0 \\
& \frac{d}{d \eta} \eta \frac{d f_{2}(\eta)}{d \eta}+\left(k^{2} \eta-\frac{\mu^{2}}{4 \eta}+21 k \chi\right) f_{2}(\eta)=0 \\
& \frac{d^{2} f_{3}(\phi)}{d \phi^{2}}+\mu^{2} f_{3}(\phi)=0,
\end{aligned}
$$

where $\chi$ and $\mu$ are arbitrary complex parameters. In the notation of Buchholz [1], the two linearly independent solutions of the first of these are:

$$
f_{1}(\xi)=m_{\chi}^{\mu}(-2 i k \xi)=\frac{(-2 i k \xi)^{\mu / 2} e^{i k \xi}{ }_{1} F_{1}\left(\frac{1+\mu}{2}-\chi ; 1+\mu ;-2 i k \xi\right)}{\Gamma(1+\mu)}
$$

where the Kummer function is defined as usual by

$$
\begin{aligned}
& { }_{1} F_{1}(a ; b ; z)=\sum_{n=0}^{\infty} \frac{(a)_{n}}{(b)_{n}} \frac{z^{n}}{n !} \\
& (a)_{n}=a(a+1) \ldots(a+n-1) \\
& (a)_{0}=1 ;
\end{aligned}
$$

and

$$
f_{1}(\xi)=w_{\chi}^{\mu}(-2 i k \xi)=\frac{\pi}{\sin \pi \mu}\left[\frac{m^{-\mu}(-2 i k \xi)}{\Gamma\left(\frac{1+\mu}{2}-\chi \chi\right)}-\frac{m^{\mu}(-2 i k \xi)}{\Gamma\left(\frac{1-\mu}{2}-\chi \chi\right)}\right] .
$$

When $\mu$ is an integer the function $w_{\chi}^{\mu}(z)$ must be derived from the above definition by a limiting process. Similarly the two linearly independent solu- 
tions of the second equation can be stated as

$$
f_{2}(\eta)=m_{x}^{\mu}(2 i k \eta)=\frac{(2 i k \eta)^{\mu / 2} e^{-i k \eta}{ }_{1} F_{1}\left(\frac{1+\mu}{2}-\chi ; 1+\mu ; 2 i k \eta\right)}{\Gamma(1+\mu)}
$$

and

$$
f_{2}(\eta)=w_{\chi}^{\mu}(2 i k \eta)=\frac{\pi}{\sin \pi \mu}\left[\frac{m_{\chi}^{-\mu}(2 i k \eta)}{\Gamma\left(\frac{1+\mu}{2}-\chi \chi\right)}-\frac{m_{\chi}^{\mu}(2 i k \eta)}{\Gamma\left(\frac{1-\mu}{2}-\chi \chi\right)}\right] .
$$

When $\mu$ is an integer, $m_{\chi}^{\mu}(z)$ is regular and single-valued over the whole space, but $W^{\mu}(z)$ is in general neither single-valued nor regular. Buchholz [1] has shown that the Green's function for the first boundary-value problem has the following integral representation in parabolic coordinates:

$$
\begin{aligned}
& G_{I}=\frac{2 i k}{4 \pi} \sum_{p=-\infty}^{\infty} e^{i p(\phi-\phi)} \frac{I}{2 \pi i} \int_{-\sigma-i \infty}^{-\sigma+i \infty} \Gamma\left(s+\frac{1+p}{2}\right) \Gamma\left(-s+\frac{1+p}{2}\right)\left|\begin{array}{ll}
m_{s}^{p}\left(-2 i k \eta_{a}\right) & w_{s}^{p}\left(-2 i k \eta_{a}\right) \\
m_{s}^{p}\left(-2 i k \eta_{0}\right) & w_{s}^{p}\left(-2 i k \eta_{0}\right)
\end{array}\right| \\
& \frac{m_{s}^{p}(-2 i k \eta)}{m_{s}^{p}\left(-2 i k \eta_{a}\right)}\left\{\begin{array}{ll}
m_{-s}^{p}(-2 i k \xi) & { }_{w}^{p}\left(-2 i k \xi_{0}\right) \\
m_{-s}^{p}\left(-2 i k \xi_{0}\right) & w_{-s}^{p}(-2 i k \xi)
\end{array}\right\} d s \quad \begin{array}{c}
\xi<\xi_{0} \\
\xi>\xi_{0}
\end{array},
\end{aligned}
$$$$
\eta_{a} \geq \eta_{0}>\eta \geq 0,0<\sigma_{p}<\frac{1+p}{2}
$$

By use of the Wronskian $[1]$

$$
w\left\{w_{s}^{p}(z), m_{s}^{p}(z)\right\}=\frac{1}{z \Gamma\left(\frac{1+p}{2}-s\right)}
$$

it is possible to write 


$$
\begin{aligned}
&\left.\frac{\partial G_{1}}{\partial \eta_{0}}\right]_{\eta_{0}}=\eta_{a}=\frac{-2 i k}{4 \pi \eta_{a}} \sum_{p^{=}-\infty}^{\infty} e^{i p\left(\phi-\phi_{0}\right)} \frac{1}{2 \pi i} \int_{-\sigma_{p}-1 \infty}^{-\sigma_{p}+1 \infty \infty} \frac{\Gamma\left(s+\frac{1+p}{2}\right) m_{s}^{p}(-2 i k \eta)}{m_{s}^{p}\left(-2 i k \eta_{a}\right)} \\
& \cdot\left\{\begin{array}{l}
m_{-s}^{p}(-2 i k \xi) w_{-s}^{p}\left(-2 i k \xi_{0}\right) \\
m_{-s}^{p}\left(-2 i k \xi_{0}\right) w_{-s}^{p}(-2 i k \xi)
\end{array}\right\} \begin{array}{r}
\xi s<\xi_{0} \\
\xi>\xi_{0}
\end{array}
\end{aligned}
$$

The integrals under the summation can be evaluated by closing the contour on the left and summing over the residues of $\Gamma\left(s+\frac{l+p}{2}\right)$. Keference to the asymptotic formulas [l] for the parabolic functions with respect to the parameter $s$ shows that this is permissible provided that

$$
\sqrt{\eta_{a}}>\sqrt{\xi}+\sqrt{\xi_{0}}+\sqrt{\eta}
$$

This leads to

$$
\begin{gathered}
\left.\frac{\partial G_{1}}{\partial \eta_{0}}\right]_{\eta_{0}=\eta_{a}}=\frac{-2 i k}{4 \pi \eta_{a}} \sum_{p=-\infty}^{\infty} e^{1 p\left(\phi-\phi_{0}\right)} \sum_{n=0}^{\infty} \frac{\Gamma(1+n+p)}{n !} m^{p}{ }_{n+\frac{1+p}{2}}(-2 i k \xi) m_{n+\frac{1+p}{2}}^{p}(2 i k \eta) \\
\\
\frac{m^{p} \frac{1+p}{2}\left(-2 i k \xi_{0}\right)}{m^{p}+\frac{1+p}{2}\left(2 i k \eta_{a}\right)} .
\end{gathered}
$$

It now follows frcm the definition of the function $m_{\chi}^{\mu}(z)$ that

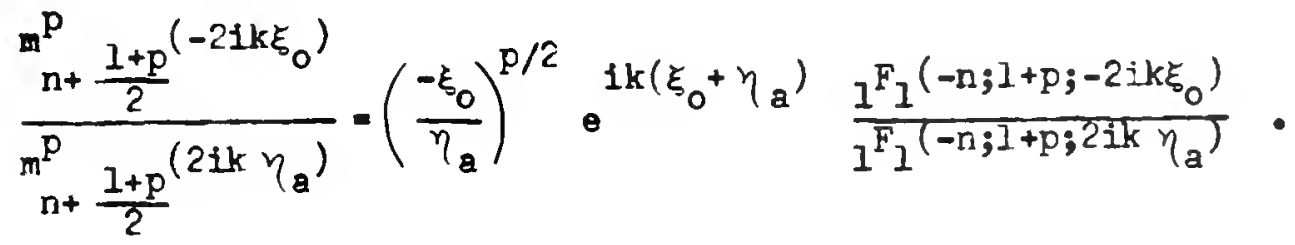


The Kumer functions appearing in the fraction are polynomials of degree $\mathrm{n}$. If it is now assumed that $k \xi_{0}$ and $k \eta_{a}$ are large, one can expand this ratio in an asymptotic series in $k \xi_{0}$ and $k \eta_{a}$. We assume for the moment, however, that although $k$ becomes large, $\xi$ and $\eta$ are such that $k \xi$ and $k \eta$ can etill be considered relatively small. Then

$$
\frac{I_{1}\left(-n ; 1+p ;-2 i k \xi_{0}\right)}{I_{1}\left(-n ; 1+p ; 2 i k \eta_{a}\right)} \approx\left(-\frac{\xi_{0}}{a}\right)^{n}
$$

Now use of the generating function $[4]$

$$
\sum_{n=0}^{\infty} \frac{\Gamma(1+n+p)}{n !} \pi_{n+\frac{1+p}{2}}^{p}(-2 i k \xi) m_{n}^{p}{ }_{\frac{1+p}{2}}(2 i k \eta)(-t)^{n}=\frac{\exp \left[i k(\xi-\eta) \frac{1-t}{1+t}\right] J_{p}\left(\frac{4 k \sqrt{\xi \eta t}}{1+t}\right)}{t^{p / 2}(1+t)}
$$

allows one to write asymptotically

$$
\left.\frac{\partial G_{1}}{\partial \eta_{0}}\right]_{\eta_{0}=\eta_{a}}=\frac{-21 k}{4 \pi \eta_{a}} \sum_{p=-\infty}^{\infty} e^{1 p\left(\phi-\phi_{0}\right)}(-)^{p / 2}
$$

$$
\frac{\exp \left(1 k\left[\left(\xi_{0}+\eta_{a}\right)+(\xi-\eta) \frac{\eta_{a}-\xi_{0}}{\eta_{a}+\xi_{0}}\right]\right) J_{p}\left(\frac{4 k \sqrt{\xi \eta \xi_{0} \eta_{a}}}{\xi_{0}+\eta_{a}}\right)}{1+\frac{\xi_{0}}{\eta_{a}}} .
$$

This expression can be simplified by use of the generating function of the Bessel function, i.e.,

$$
\sum_{p=-\infty}^{\infty} J_{p}(z) t^{p}=\exp \left[z\left(t-\frac{1}{t}\right) / 2\right] ;
$$

than one obtains

$\left.\frac{\partial G_{1}}{\partial \eta_{0}}\right]_{\eta_{0}=\eta_{a}}=\frac{-2 i k}{4 \pi\left(\eta_{a}+\xi_{0}\right)} \exp \left(1 k\left[\eta_{a}+\xi_{0}+(\xi-\eta) \frac{\eta_{a}-\xi_{0}}{\eta_{a}+\xi_{0}}-\frac{4 \sqrt{\xi \eta \xi_{0} \eta_{a}}}{\eta_{a}+\xi_{0}} \cos \left(\phi-\phi_{0}\right)\right]\right)$. 
The distance between two pointe in parabolic coordinates is given by the expression

$$
r=\sqrt{\left(\eta_{a}+\xi_{0}\right)^{2}+(\eta+\xi)^{2}+2(\xi-\eta)\left(\eta_{a}-\xi_{0}\right)-8 \sqrt{\xi \eta \xi_{0} \eta_{a}} \cos \left(\phi-\phi_{0}\right)}
$$

which, under the assumption that the quantities $\eta_{a}, \xi_{0}$ are large relative to $\eta, \xi$, can be expanded in a binomial series. Use of wat series shows that one can now write

$$
\left.\frac{\partial G_{1}}{\partial \eta_{0}}\right]_{\eta_{0}=\eta_{a}}=\frac{-21 k}{4 \pi} \frac{e^{1 k r}}{r}
$$

and inserting this result in the expression

$$
u=\iint \bar{u} \frac{\partial G_{1}}{\partial \mathbb{N}} d \theta
$$

leads to

$$
\left.u=2 \eta_{a} \iint \bar{u} \frac{\partial G}{\partial \eta_{0}}\right]_{\eta_{0}=\eta_{a}} d \xi d \phi=\frac{-i k \eta_{a}}{\pi} \int_{0}^{\infty} \int_{0}^{2 \pi} \bar{u} \frac{e^{i k r}}{r} d \xi d \phi .
$$

In a similar manner it is possible to start with the integral representation of the Green's function for the second boundary-value problem to derive an asymptotic representation. Then it follows from

$$
\begin{aligned}
& G_{2}=\frac{-2 i k}{4 \pi} \sum_{p=-\infty}^{\infty} e^{i p\left(\phi-\phi_{0}\right)} \frac{1}{2 \pi I} \int_{-\sigma_{p}-i \infty}^{-\sigma_{p}+i \infty} \Gamma\left(s+\frac{1+p}{2}\right) \Gamma\left(-s+\frac{1+p}{2}\right) m_{s}^{p}(-2 i k \xi) w_{s}^{p}\left(-2 i k \xi_{0}\right)
\end{aligned}
$$

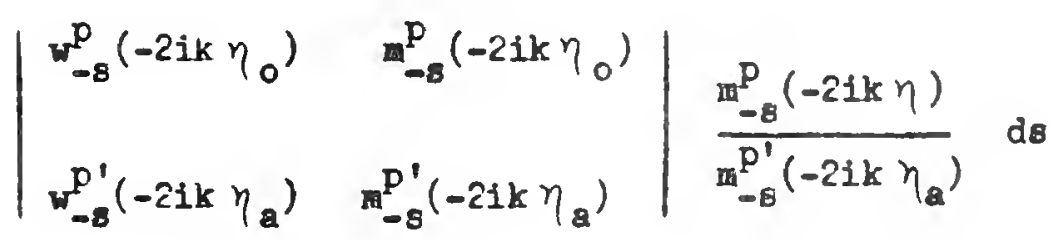


$-\mathfrak{H}-$

$$
G_{2}=\frac{1}{2 \pi} \frac{e^{i k r}}{r}
$$

and

$$
\left.u=\iint G_{2} \frac{\partial \bar{u}}{\partial N} d s=\frac{\eta_{a}}{\pi} \int_{0}^{\infty} \int_{0}^{2 \pi} \frac{\partial \bar{u}}{\partial \eta}\right]_{\eta=\eta_{a}}^{\frac{e^{i k r}}{r}} d \xi d \phi .
$$

4. The Green'e function in parabolic cylinder coordinates

The parabolic cylinder coordinate system can be defined by the transformation

$$
\begin{aligned}
& x=\xi-\eta \\
& y= \pm 2 \sqrt{\xi \eta} \\
& \rho=\sqrt{x^{2}+y^{2}}=\xi+\eta \\
& x=\rho \cos \phi \quad y=\rho \sin \phi .
\end{aligned}
$$

As in the previous case the surfaces $\xi=$ constant and $\eta=$ constant are parabolas. The save equation

$$
\Delta u+k^{2} u=0
$$

when expressed in parabolic cylinder coordinates becomes

$$
\frac{1}{4(\xi+\eta)}\left\{\frac{\partial^{2} u}{\partial(\sqrt{\xi})^{2}}+\frac{\partial^{2} u}{\partial(\sqrt{\eta})^{2}}\right\}+k^{2} u=0 .
$$

Separation of variables leads to the two ordinary differential equation e

$$
\begin{aligned}
& f_{1}^{\prime \prime}(\sqrt{\xi})+\left[(2 k \sqrt{\xi})^{2}+4 i k\left(r+\frac{1}{2}\right)\right] f_{1}(\sqrt{\xi})=0 \\
& f_{2}^{\prime \prime}(\sqrt{\eta})+\left[(2 k \sqrt{\eta})^{2}-4 i k\left(\gamma+\frac{1}{2}\right)\right] f_{2}(\sqrt{\eta})=0,
\end{aligned}
$$


where $Y$ is an arbitrary complex parameter. Two linearly independent solutions to the first of these can be defined by

$$
\begin{aligned}
& \mathrm{E}_{\gamma}^{(0)}(2 \sqrt{-1 \mathrm{k \xi}})=\sqrt{2 \pi}(-21 k \xi)^{1 / 4} \mathrm{~m}_{\frac{\gamma}{2}}^{-1 / 2}+\frac{1}{4}(-\hat{\alpha} i k \xi), \\
& E_{Y}^{(1)}(2 \sqrt{-1 k \xi}) \cdot \sqrt{2 \pi}(-2 i k \xi)^{1 / 4} \frac{m^{m}}{\frac{\gamma}{2}+\frac{1}{4}}(-2 i k \xi) .
\end{aligned}
$$

Another solution which proves convenient can be expressed in terms of these two a8

$$
\begin{aligned}
D_{Y}(2 \sqrt{-i k \xi}) & =2^{Y / 2} \sqrt{\pi / 2}\left\{\frac{E_{Y}^{(0)}(2 \sqrt{-1 k \xi})}{\Gamma\left(\frac{1-\gamma}{2}\right)}-\frac{E_{Y}^{(1)}(2 \sqrt{-i k \xi})}{\Gamma\left(-\frac{\gamma}{2}\right)}\right\} \\
& =2^{Y / 2}(-\hat{i} i k \xi)^{1 / 4} w_{\frac{\gamma}{2}+\frac{1}{4}}^{1 / 2}(-\hat{c} i k \xi),
\end{aligned}
$$

where the functions $\operatorname{mon}_{\chi}^{\mu}(z)$ and $w_{\chi}^{\mu}(z)$ were defined in the previous section. Similarly the solutions of the second equation can be written as

$$
\begin{aligned}
& E_{Y}^{(0)}(2 \sqrt{i k \eta})=\sqrt{2 \pi}(2 i k \eta)^{1 / 4} m_{\frac{r}{2}+\frac{1}{4}}^{-1 / 2}(2 i k \eta), \\
& \mathrm{E}_{Y}^{(1)}(2 \sqrt{i \mathrm{k} \eta})=\sqrt{2 \pi}(2 i k \eta)^{1 / 4} \mathrm{~m}_{\frac{\gamma}{2}+\frac{1}{4}}^{+1 / 2}(2 i k \eta), \\
& D_{\gamma}(2 \sqrt{1 k \eta})=2^{\gamma / 2} \sqrt{\pi / 2}\left\{\frac{E_{\gamma}^{(0)}(2 \sqrt{1 k \eta})}{\Gamma\left(\frac{1-\gamma}{2}\right)}-\frac{E_{\gamma}^{(1)}(2 \sqrt{1 k \eta})}{\Gamma\left(-\frac{\gamma}{2}\right)}\right\} \\
& =2^{\gamma / 2}(21 k \eta)^{1 / 4} \frac{\gamma}{\frac{\gamma}{2}+\frac{1}{4}}(2 i k \eta) .
\end{aligned}
$$


Buchholz [] has shown that it is possible to represent the Hankel functions of the first as well as the second kind in terms of these functions. The former can be expressed by

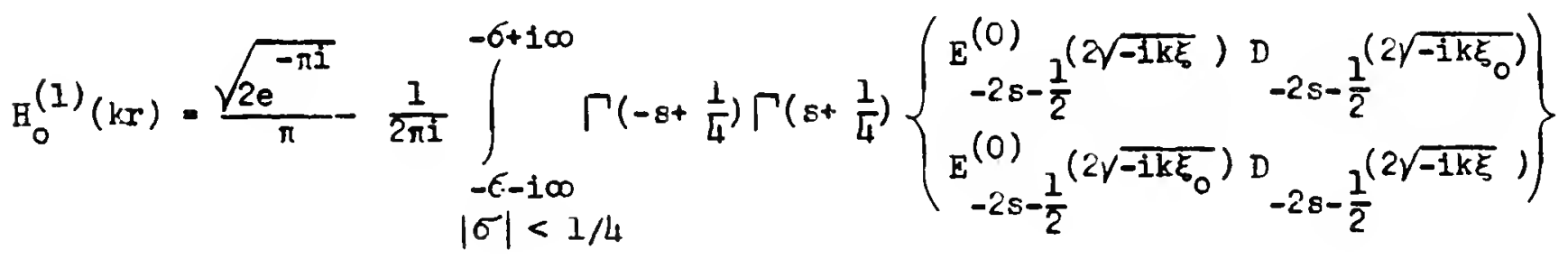

$$
\left\{\begin{array}{ll}
\mathrm{E}^{(0)}{ }_{2 s-\frac{1}{2}}^{(2 \sqrt{-1 \mathrm{k} \eta})} & D_{2 s-\frac{1}{2}}^{\left(2 \sqrt{-i k \eta_{0}}\right)} \\
\mathrm{E}^{(0)}\left(2 \sqrt{-i k \eta_{0}}\right) & D_{2 s-\frac{1}{2}}^{(2 \sqrt{-i k \eta})}
\end{array}\right\} \mathrm{ds}
$$

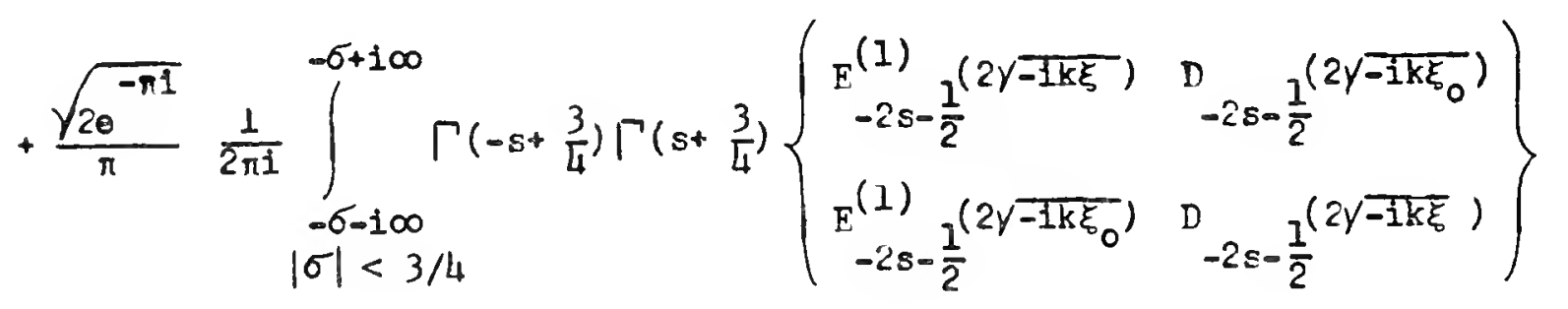

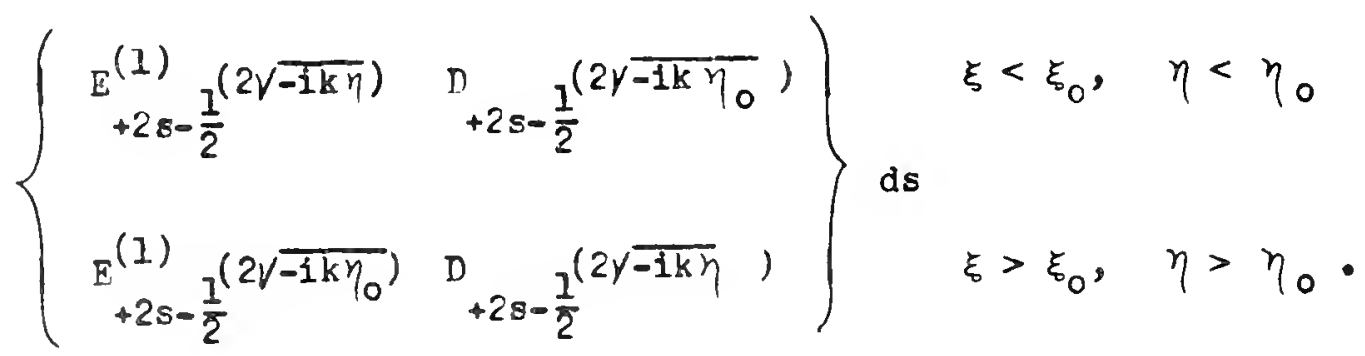

With this integral representation it is now possible to construct the Green's functions for the first as well as the second boundary-value problems. We obtain 
in the case of the first boundary-value problem

$$
\begin{aligned}
& G_{1}=\frac{\sqrt{2 e^{-\pi I}}}{\pi} \frac{1}{2 \pi I} \int_{\substack{-\sigma-1 \infty \\
|\sigma|<1 / 4}}^{-\sigma+1 \infty} \Gamma\left(-s+\frac{1}{4}\right) \Gamma\left(s+\frac{1}{4}\right) E_{-2 s-\frac{1}{2}}^{(0)}(2 \sqrt{-i k \xi}) D_{-2 s-\frac{1}{2}}\left(2 \sqrt{-1 k \xi_{0}}\right)
\end{aligned}
$$

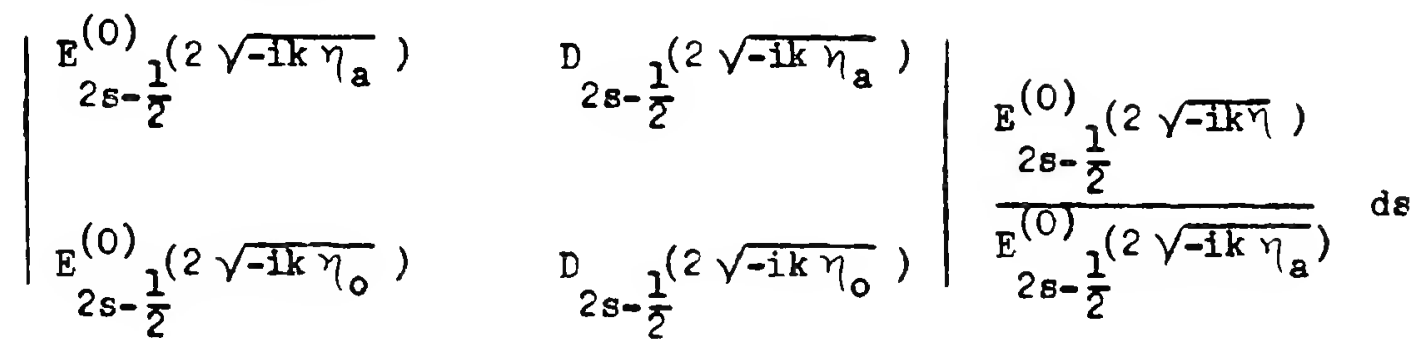$$
\left.+\frac{\sqrt{2 e^{-\pi i}}}{\pi} \frac{1}{2 \pi i} \int_{\substack{-\sigma-1 \infty \\|\sigma|<3 / 4}}^{-\sigma+100} \Gamma\left(-s+\frac{3}{4}\right) \Gamma\left(s+\frac{3}{4}\right) \mathrm{E}_{-2 s-\frac{1}{2}}^{(1)}(2 \sqrt{-1 k \xi}) D_{-2 s-\frac{1}{2}}^{\left(2 \sqrt{-1 k \xi_{0}}\right.}\right)
$$

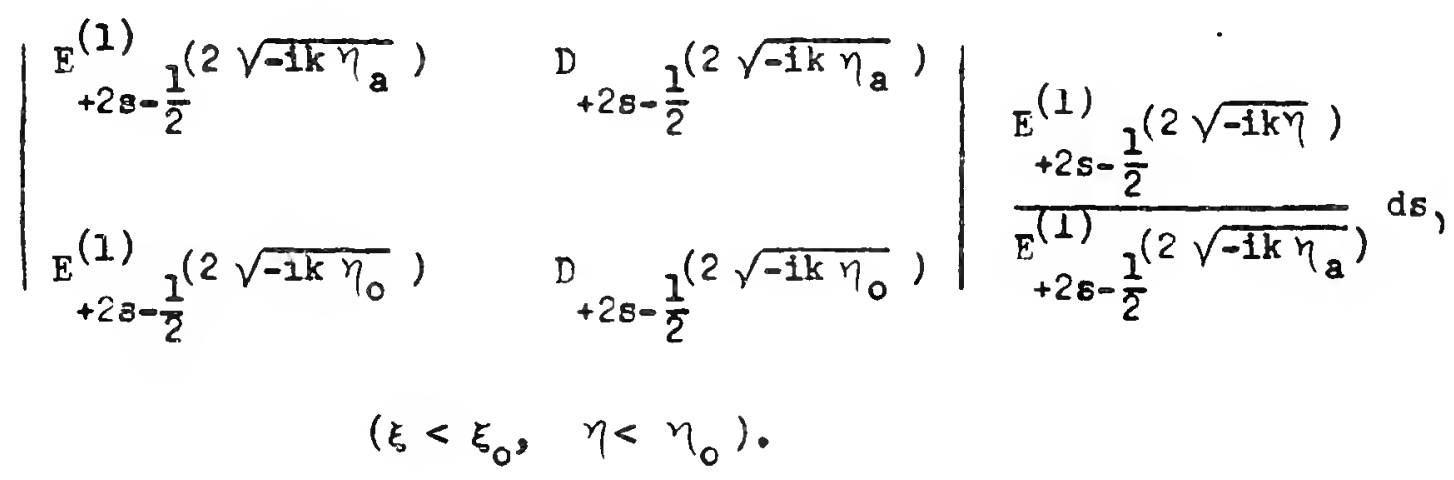

Use of the Wronskian [1]

$$
\begin{aligned}
& W\left\{D_{\gamma}(z), E_{Y}^{(0)}(z)\right\}=\sqrt{\pi} \frac{2^{1+\varphi / 2}}{\Gamma\left(-\frac{\gamma}{2}\right)} \\
& W\left\{D_{\gamma}(z), E_{\gamma}^{(1)}(z)\right\}=\sqrt{\pi} \frac{2^{1+\varphi / 2}}{\Gamma\left(\frac{1-\gamma}{2}\right)}
\end{aligned}
$$


allows one to calculate

$$
\begin{aligned}
& \left.\frac{\partial G_{1}}{\partial \eta_{b}}\right]_{\eta_{b}=\eta_{a}}=\sqrt{\frac{i k 2^{5 / 2}}{\pi \eta_{a}}} \frac{1}{2 \pi I} \int_{\substack{-\sigma-i \infty \infty \\
1 \sigma<1 / 4}}^{-\sigma+i \infty 0} 2^{8} \Gamma\left(s+\frac{1}{4}\right) E_{-2 s-\frac{1}{2}}^{(0)}(2 \sqrt{-1 k \xi}) D_{-2 s-\frac{1}{2}}^{\left(2 \sqrt{-1 k \xi_{0}}\right)} \\
& \underbrace{E^{(0)}}_{2 s-\frac{1}{2}} \frac{1}{\left(2 \sqrt{-1 k \eta_{a}}\right)} d s
\end{aligned}
$$

$-\sigma+i 00$

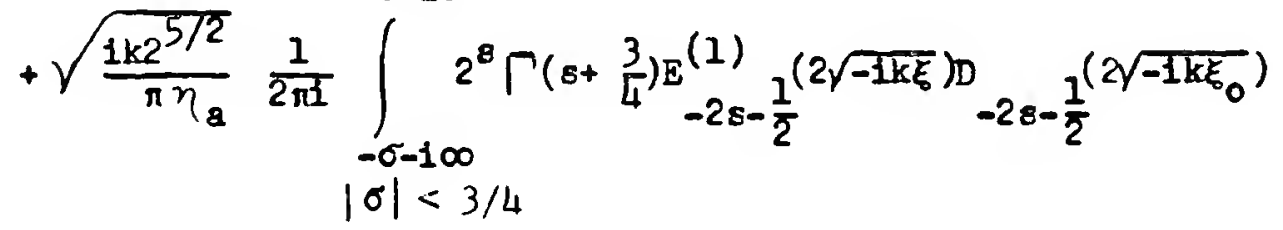

$$
\frac{E^{(1)}(2 \sqrt{-1 \mathrm{k} \eta})}{28-\frac{1}{2}} \mathrm{E}_{28-\frac{1}{2}}^{\left.(1) \sqrt{-1 \mathrm{k} \eta_{\mathrm{a}}}\right)} \text { ds, }\left(\xi<\xi_{0}\right) \text {. }
$$

Wherever wo have

$$
\sqrt{\eta_{a}}>\sqrt{\xi}+\sqrt{\xi_{0}}+\sqrt{\eta}
$$

we can close the contour on the left and evaluate the above integrals by suming over the residues of the gamma functions:

$$
\begin{aligned}
&\left.\frac{\partial G_{1}}{\partial \eta_{0}}\right]_{\eta_{0}=\eta_{a}}=\sqrt{\frac{i k 2^{5 / 2}}{\pi \eta_{a}}}\left\{\sum_{N=0}^{\infty} \frac{2^{-N-\frac{1}{4}}(-)^{N}}{N !} E_{2 N}^{(0)}(2 \sqrt{-1 k \xi}) D_{2 N}\left(2 \sqrt{-1 k \xi_{0}}\right) \frac{E^{(0)}}{E_{-2 N-1}^{(0)}(2 \sqrt{-1 k \eta})}\right. \\
&\left.+\sum_{N=0}^{\infty} \frac{\left.2^{-N-\frac{3}{4}\left(-1 k \eta^{N}\right.}\right)}{N !} F_{2 N+1}^{(1)}(2 \sqrt{-1 k \xi}) D_{2 N+1}\left(2 \sqrt{-1 k \xi_{0}}\right) \frac{E_{-2 N-2}^{(1)}(2 \sqrt{-1 k \eta})}{E_{-2 N-2}^{(1)}\left(2 \sqrt{-1 k \eta_{a}}\right)}\right\} .
\end{aligned}
$$


By use of the functions $m_{\chi}^{\mu}(z)$ and $w_{x}^{\mu}(z)$ it 18 now possible to rewrite this expression in a form similar to the one arrived at in the case of the paraboloid of revolution:

$$
\begin{aligned}
& \left.\frac{\partial G_{1}}{\partial \eta_{0}}\right]_{\eta_{0}=\eta_{a}}=\frac{-4 k}{\sqrt{\eta_{a}}}\left(\frac{\xi \eta \xi_{0}}{\eta_{a}}\right)^{1 / 4}\left\{\sum_{N=0}^{\infty} \frac{\Gamma\left(N+\frac{1}{2}\right)}{N !} m^{-1 / 2}(-2 i k \xi) m^{-1 / 2}(2 i k \eta)\right. \\
& \frac{m^{-1 / 2}\left(-2 i k \xi_{0}\right)}{m^{-1 / 2}\left(2 i k \eta_{a}\right)} \\
& \left.+\sum_{N=0}^{\infty} \frac{\Gamma\left(N+\frac{3}{2}\right)}{N !} m_{N=\frac{3}{4}}^{1 / 2}(-2 i k \xi) m_{N+\frac{3}{4}}^{1 / 2}(2 i k \eta) \frac{m^{1 / 2}\left(-2 i k \xi_{0}\right)}{m^{1 / 2}\left(2 i k \eta_{a}\right)}\right\} \text {. }
\end{aligned}
$$

If it is now assumed that $k \xi_{0}$ and $k \eta_{a}$ are large relative to $k \xi_{0}$ and $k \eta$ one can take advantage of the asymptotic relations

$$
\frac{m^{ \pm 1 / 2}+\frac{1}{2} \pm \frac{1}{4}\left(-2 i k \xi_{0}\right)}{m^{ \pm 1 / 2}}=e^{i k\left(\xi_{0}+\eta_{a}\right)}\left(-\frac{1}{2} \bar{\eta}_{0}\right)^{N \pm 1 / 4}
$$

and then by an aplication of the generating function of the confluent hypergeomotric functions $[4]$ one obtains

$$
\begin{aligned}
& \left.\frac{\partial G_{1}}{\partial \eta_{0}}\right]_{\eta_{0}=\eta_{a}}=\frac{-4 k\left(\xi \eta \xi_{0} \eta_{a}\right)^{\frac{1}{4}}}{\eta_{a}+\xi_{0}}\left\{\operatorname { e x p } \left[i k ( \eta _ { a } + \xi _ { 0 } + ( \xi - \eta ) \frac { \eta _ { a } - \xi _ { 0 } } { \eta _ { a } + \xi _ { 0 } } ] \left[e_{-}^{+\pi i / \mu_{J}}\left(\frac{4 k \sqrt{\xi \eta \xi_{0}} \eta_{a}}{\eta_{a}+\xi_{0}}\right)\right.\right.\right. \\
& \left.\left.\left.+e^{-\pi i / J_{1}} \frac{4 k \sqrt{\xi \eta \xi_{0} \eta_{a}}}{\eta_{a}+\xi_{0}}\right)\right]\right\} \\
& =\sqrt{\frac{+8 i k}{\pi\left(\eta_{a}+\xi_{0}\right)}} \exp \left(1 k\left[\left(\eta_{a}+\xi_{0}\right)+(\xi-\eta) \frac{\eta_{a}-\xi_{0}}{\eta_{a}+\xi_{0}}-\frac{4 \sqrt{\xi \eta \xi_{0} \eta_{a}}}{\eta_{a}+\xi_{0}}\right]\right) \text {. }
\end{aligned}
$$


The distance $r$ betwen two points can be expanded into a rapidly converging binomial sories if $\xi_{0}$ and $\eta_{a}$ are large compared to $\xi$ and $\eta$ :

$$
\begin{aligned}
r & =\sqrt{\left(\eta_{a}+\xi_{0}\right)^{2}+(\eta+\xi)^{2}+2(\xi-\eta)\left(\eta_{a}-\xi_{0}\right)-8 \sqrt{\xi \eta \xi_{0} \eta_{a}}} \\
& =\eta_{a}+\xi_{0}+(\xi+\eta) \frac{\eta_{a}-\xi_{0}}{\eta_{a}+\xi_{0}}-\frac{4 \sqrt{\xi \eta \xi_{0} \eta_{a}}}{\eta_{a}+\xi_{0}} .
\end{aligned}
$$

Hence one can replace the preceding expression by

$$
\left.\frac{\partial G_{1}}{\partial \eta_{0}}\right]_{\eta_{0}}=\eta_{a}=\sqrt{\frac{+8 i k}{\pi r}} e^{i k r}
$$

and since this is an asymptotic expression it could be written as

$$
\left.\frac{\partial g_{1}}{\partial \eta_{0}}\right]_{\eta_{0}}=\eta_{a}=2 i k H_{0}^{(1)}(\mathrm{kr})
$$

One can now write by means of Green's theorem

$u=\frac{2}{4 I} \int \bar{u} \frac{\partial G_{1}}{\partial N^{\prime}} d s=\frac{1}{4 I} \int \bar{u} \frac{\partial G_{1}}{\partial \eta} \prod_{\eta_{0}=\eta_{a}} \sqrt{\frac{\eta_{a}}{\xi}} d \xi=2 \sqrt{\frac{-21 k \eta_{a}}{\pi}} \int_{-\infty}^{\infty} \bar{u} \frac{e^{i k r}}{\sqrt{r}} d \sqrt{\xi}$. The above integrals must be taken over the whole reflector, which falls into two parts defined by

$$
\sqrt{\eta}=\sqrt{\eta_{a}}, \sqrt{\xi}>0 \text { and } \sqrt{\xi}<0
$$

A process similar to the one just carried out leads to an asymptotic representation of the Green's function for boundary-value probleme of the second kind. Then 
$-18-$

$$
\begin{aligned}
& G_{2}=\frac{\sqrt{2 e^{-\pi I}}}{\pi} \frac{1}{2 \pi} \int_{\substack{-\sigma-i \infty \\
|\sigma|<1 / 4}}^{-\sigma+i \infty} \Gamma\left(-s+\frac{1}{4}\right) \Gamma\left(s+\frac{1}{4}\right) E_{-28-\frac{1}{2}}^{(0)}(2 \sqrt{-i k \xi}) ! \sum_{-28-\frac{1}{2}}\left(2 \sqrt{-i k \xi_{0}}\right)
\end{aligned}
$$

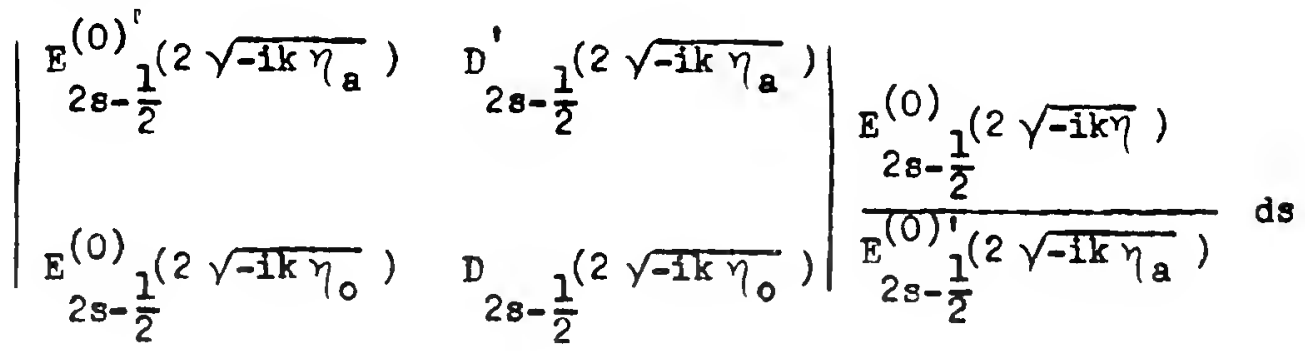

$$
\begin{aligned}
& +\frac{\sqrt{2 e^{-\pi I}}}{\pi} \frac{1}{2 \pi I} \int_{\substack{-\sigma-i \infty \\
|\sigma|<3 / 4}}^{-\sigma+i \infty} \Gamma\left(-s+\frac{3}{4}\right) \Gamma\left(s+\frac{3}{4}\right) E_{-2 s-\frac{1}{2}}^{(1)}{ }^{(2 \sqrt{-i k \xi})} D_{-2 s-\frac{1}{2}}\left(2 \sqrt{-i k \xi_{0}}\right)
\end{aligned}
$$

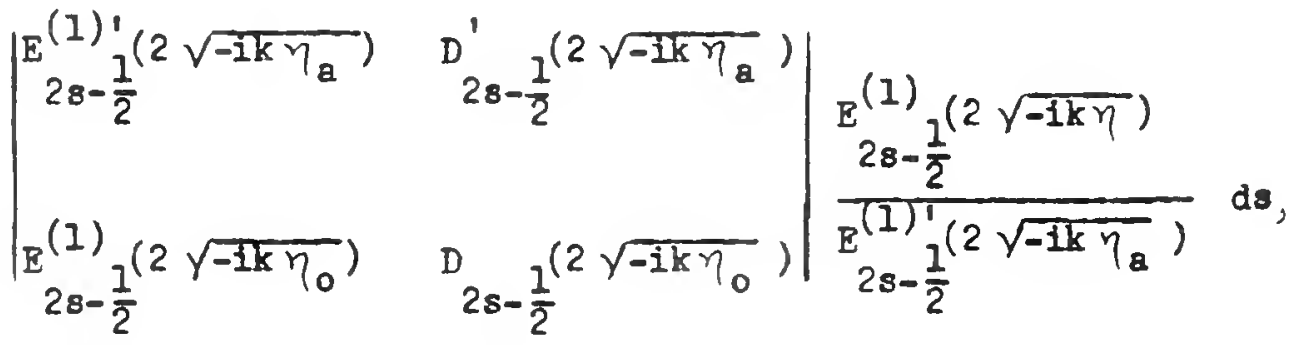

$$
\begin{aligned}
& \left(\xi<\xi_{0}, \quad \eta<\eta_{0}\right),
\end{aligned}
$$

from which one can show by a similar procedure that asymptotically

$$
G_{2}=2 \frac{e^{i k\left(r-\frac{\pi}{4}\right)}}{\sqrt{\pi k r}}-\sqrt{2} H_{0}^{(1)}(k r) .
$$

Then it follows that

$$
u=\frac{1}{4} \int G_{2} \frac{\partial \bar{u}}{\partial \bar{N}} d s=\frac{1}{4} \int \frac{2 e^{1 k\left(r-\frac{\pi}{4}\right)}}{\sqrt{\pi k r}} \frac{\partial \bar{u}}{\partial \eta} \sqrt{\frac{\eta a}{\xi}} d \xi=\frac{1 e^{-i \frac{\pi}{4}} \sqrt{\eta} a}{\sqrt{\pi k}} \int_{-\infty}^{\infty} \frac{e^{i k r}}{\sqrt{r}} \frac{\partial \bar{u}}{\partial \eta} d \sqrt{\xi} .
$$


5. Applications of the asymptotic formulas to diffraction problems

We will now apply the previously derived formulas to numerous cases.

a). First we shall treat the diffraction of a plane wave by a paraboloidal reflector. Then, taking

$$
\bar{u}=e^{-i k\left(\xi-\eta_{a}\right)}
$$

we have

$$
u=+\frac{i k \eta_{a}}{\pi} \int_{0}^{\infty} \int_{0}^{2 \pi} \frac{e^{i k\left[R-\xi+\eta_{a}\right]}}{R} d \xi d \phi
$$

where

$$
R=\sqrt{\left(\xi-\eta_{a}-\xi_{0}+\eta\right)^{2}+4 \xi \eta_{a}+4 \xi_{0} \eta-8 \sqrt{\xi \xi_{0} \eta_{\eta_{a}}} \cos \phi}
$$

so that u is evaluated at the point

$$
\begin{aligned}
& x=2 \sqrt{\xi_{0} \eta} \\
& y=0 \\
& z=\xi_{0}-\eta
\end{aligned}
$$

and the general point on the paraboloid is given by

$$
\begin{aligned}
& x_{a}=2 \sqrt{\xi \eta_{a}} \cos \phi \\
& y_{a}=2 \sqrt{\xi \eta_{a}} \sin \phi \\
& z_{a}=\xi-\eta_{a} .
\end{aligned}
$$

Two points of stationary phase can be found. Both lie in the plane $y_{a}=0$ and are given by the intersections of the parabola and the line

$$
\frac{x_{a}}{z_{a}}=\frac{x}{z}
$$




$$
\frac{\xi \eta_{a}}{\left(\xi-\eta_{a}\right)^{2}}=\frac{\xi_{0} \eta}{\left(\xi_{0}-\eta\right)^{2}}
$$

Denote the root corresponding to $q=\pi$ by $\xi_{1}$ and the other by $\xi_{2}$. Then near these roots one can use the expansions

$$
R-\xi=R_{1}-\xi_{1}-\frac{\left(\xi-\xi_{1}\right)^{2}}{2} \sqrt{\frac{\xi_{0} \eta \eta_{a}}{\xi_{1}^{3} R_{1}^{2}}}-(\phi-\pi)^{2} \frac{2 \sqrt{\xi_{1} \xi_{0} \eta \eta_{a}}}{R_{1}}
$$

near $\xi_{1}$ and

$$
R-\xi=R_{2}-\xi_{2}+\frac{\left(\xi-\xi_{2}\right)^{2}}{2} \sqrt{\frac{\xi_{0} \eta_{a}}{\xi_{2}^{3} R_{2}^{2}}}+\phi^{2} \frac{2 \sqrt{\xi_{2} \xi_{0} \eta \eta_{a}}}{R_{2}}
$$

near $\xi_{2}$. It is now possible to write the solution by an application of the method of stationary phase. We have

$$
u=\sqrt{\frac{\eta_{a} \xi_{1}}{\xi_{0} \eta}} e^{1 k\left(R_{1}-\xi_{1}+\eta_{a}\right)}-\sqrt{\frac{\eta_{a} \xi_{2}}{\xi_{0} \eta}} e^{1 k\left(R_{2}-\xi_{2}+\eta_{a}\right)} .
$$

The above solution is valid only if the coordinate $x$ does not vanish. If it does there is no true point of stationary phase. However, one can still evaluate the integral asymptotically in this case. Then

$$
R=\sqrt{\left(\xi-\eta_{a}-\xi_{0}\right)^{2}+4 \xi \eta_{a}}
$$

is independent of $\varnothing$, so that

$$
u=-2 i k \eta_{a} e^{i k \eta} \int_{0}^{\infty} \frac{e^{i k(R-\xi)}}{R} d \xi \text {. }
$$

If we let $x=k \xi$ under the integral, use binomial series, and drop higher-order terms we obtain directly

$$
u=\frac{e^{i k\left(2 \eta_{a}+\xi_{0}\right)}}{\xi_{0}}
$$


This analysis shows that the diffracted wave has the character of a point source near the focus.

An analogous procedure can be followed with the parabolic cylinder, and one obtains

$$
u=+4 i\left(\frac{\eta_{a} \xi_{1}}{\xi_{0} \eta}\right)^{\frac{1}{4}} e^{i k\left(R_{1}-\xi_{1}+\eta_{a}\right)}-4\left(\frac{\eta_{a} \xi_{2}}{\xi_{0} \eta}\right)^{\frac{1}{4}} e^{i k\left(R_{2}-\xi_{2}+\eta_{a}\right)}
$$

for off-axis points. On the axis for $\eta=0$ one obtains

$$
u=2 i \sqrt{\frac{\eta_{a}}{\xi_{0}}} e^{i k\left(2 \eta_{a}+\xi_{0}\right)} .
$$

b). We will now consider the problem of a source at the focus of the reflector which results in an outgoing plane wave. This problem can be considered as a reciprocal problem to the problem considered above, where an incoming plane wave results in a singularity at the focus. One proceeds, as before, with the formula

$$
u=\frac{i k \eta_{a}}{\pi} \int_{0}^{\infty} \int_{0}^{2 \pi} \frac{e^{i k(R+r)}}{R r} d \xi d \phi
$$

where

$$
\begin{gathered}
\bar{u}=-\frac{e^{i k r}}{r}, \\
R=\sqrt{\left(\xi_{0}-\eta-\xi+\eta_{a}\right)^{2}+4 \xi_{o} \eta+4 \eta_{a}-8 \sqrt{\xi \xi \eta_{0} \eta_{a}} \cos \phi}, \\
r=\xi_{a}+\eta_{a} .
\end{gathered}
$$

The point at which the expression $R+r$ is stationary can be determined by geometrical optics and is given by

$$
\begin{aligned}
& \xi=\xi_{0} \frac{\eta}{\eta_{a}} \\
& \phi=0 .
\end{aligned}
$$


Expanding $R+r$ about that point leads to the following expression for the solution:

$$
\begin{aligned}
& u=\frac{i k \eta_{a}^{3} e^{i k\left(2 \eta_{a}+\xi_{0}-\eta\right)}}{\pi\left(\eta_{a}-\eta\right)\left(\eta_{a}+\xi_{0}\right)\left(\eta_{a}^{2}+\xi_{0} \eta\right)} \int_{-\infty}^{\infty} \exp i k\left[\frac{\eta_{a}^{3} \xi^{2}}{\left.2 \xi_{0} \eta^{(\eta} \eta_{a}-\eta\right)\left(\eta_{a}+\xi_{0}\right)}\right] d \xi \\
& u=\frac{-\eta_{a}}{\eta_{a}^{2}+\xi_{0} \eta} \exp \left(i k\left[2 \eta_{a}+\xi_{0}-\eta\right]\right),
\end{aligned}
$$

which is similar in character to an outgoing plane wave, but is decreasing in amplitude.

An analogous operation with the integral

$$
u=\frac{e^{-i \frac{\pi}{2}} 4 \sqrt{\eta_{a}}}{\pi} \int_{-\infty}^{\infty} \frac{e^{i k(R+r)}}{\sqrt{R r}} d \sqrt{\xi}
$$

leads to the function

$$
u=\frac{2 \sqrt{2} e^{-1 \frac{\pi}{2} e^{i k\left(2 \eta_{a}+\xi_{0}-\eta\right)}}}{\pi \sqrt{k\left(\eta_{a}+\frac{\xi_{0} \eta}{\eta_{a}}\right)}}
$$

which is the corresponding solution for a line source placed at the focus of a parabolic cylinder.

c). In this aection we shall consider the case where a point source is placed on the axis of the reflector, not at the focus, but at a distance from it. As before, we take as our starting point the formula

$$
u=-\frac{i k \eta_{a}}{\pi} \int_{0}^{\infty} \int_{0}^{2 \pi} \bar{u} \frac{e^{i k r}}{r} d \xi d \phi,
$$


where

$$
\begin{aligned}
\bar{u} & =-\frac{e^{i k R}}{R}, \\
R & =\sqrt{\left(\xi-\eta_{a}-a\right)^{2}+4 \xi \eta_{a}} \\
r & =\sqrt{\left(\xi-\eta_{a}-\xi_{0}+\eta\right)^{2}+4 \xi \eta_{a}+4 \xi \eta_{-}-8 \sqrt{\xi \xi_{0} \eta_{a}} \cos \phi} .
\end{aligned}
$$

The point given by

$$
x=2 \sqrt{\xi_{0} \eta}, \quad y=0, \quad z=\xi_{0}-\eta
$$

is the point under examination;

$$
x=2 \sqrt{\xi \eta_{a}} \cos \phi, y=2 \sqrt{\xi \eta_{a}} \sin \phi, \quad z=\xi-\eta_{a}
$$

is a general point on the surface of the paraboloid; and the source is located at

$$
x=0, \quad y=0, \quad z=a
$$

Unless the point under examination lies on the axis of the paraboloid or the caustic surface, the function

$$
u=0(1)
$$

This follows from simple stationary phase considerations and the fact that $R+r$ is such that

$$
\frac{\partial^{2}(R+r)}{\partial \xi^{2}} \neq 0 \quad \frac{\partial^{2}(R+r)}{\partial \gamma^{2}} \neq 0
$$

on the surface, but at some points

$$
\frac{\partial(R+r)}{\partial \xi}=0 \quad \frac{\partial(R+r)}{\partial \phi}=0
$$


simultaneously.

If $\eta=0$, the point under examination lies on the axis. In this case the $\theta$ integration is trivial and our formula roduces to

$$
u=21 k \eta_{a} \int_{0}^{\infty} \frac{e^{1 k(R+r)}}{R r} d \xi \text {. }
$$

The expresaion $R+r$ becomes stationary at

$$
\xi=a-\eta_{a}-\sqrt{\frac{a}{\xi_{0}}}\left(\eta_{a}-\xi_{0}\right) \text {. }
$$

But $\xi \geq 0$ if and only if $\xi_{0} \geq \eta_{a}^{2} / a$, so that we have a point of stationary phase only if the point under inspection lies above the cusp of the caustic. Then the integral can be evaluated by stationary phase techniques and one obtains

$$
u-\frac{2 \sqrt{\pi k \eta_{a}} e^{i k(\bar{R}+\bar{r})}}{\sqrt{a \bar{r}^{2} / \bar{R}+\xi_{0} \bar{R}^{2} / \bar{r}}} .
$$

Here $\bar{k}$ and $\bar{r}$ represent the values of $R$ and $r$ at the point of stationary phase. It may be observed that $u$ is on the order of $\sqrt{k}$ and remains finite even at the cusp of the caustic. For large values of a the formula reduces to

$$
u=-2 \sqrt{\frac{\pi k}{a}} e^{1 k(a+2 \eta a)}
$$

at the cusp of the caustic.

For $\xi_{0}<\eta_{a}^{2} / a$ a different technique must be employed to evaluate the integral, since no point of stationary phase exists. We replace $\xi$ by $x / k$ under the integral and simplify the integrand by dropping all terms of order $1 / \mathrm{k}$ and higher. Then the integral can be evaluated explicitly and we obtain

$$
u=\frac{-\eta_{a} e^{i k\left(2 \eta_{a}+a+\xi_{0}\right)}}{\eta_{e}^{2}-a \xi_{0}}
$$


Here $i$ is of order 1 with respect to $k$ and hes a simple pole at the cusp of the caustic, which is completely different from the situation when the cusp is approached from the opposite side.

still a different kind of behavior is observed on the caustic and at the cusp when the cusp is approached along the caustic.

If we now choose $\xi_{0}$ and $\eta$ go that they lie on the caustic surface we have

$$
u=\frac{i k \eta_{a}}{\pi} \int_{0}^{\infty} \int_{0}^{2 \pi} \frac{e^{i k(R+r)}}{R r} d \xi d \phi
$$

where

$$
\begin{gathered}
R=\sqrt{\left(\xi-\eta_{a}-a\right)^{2}+4 \xi \eta_{a}}, \\
r=\sqrt{\left(\xi-\eta_{a}-\xi_{0}+\eta\right)^{2}+4 \xi \eta_{a}+4 \xi_{0} \eta-8 \sqrt{\xi \xi_{0} \eta_{a}} \cos \phi}, \\
\frac{\partial(R+r)}{\partial \phi}=\frac{4 \sqrt{\xi \xi_{0} \eta \eta_{a}}}{r} \sin \phi=0 \quad \text { if } \phi=0, \pi .
\end{gathered}
$$

For $\phi=0$ and $\pi$ we can also find points of stationary phase such that

$$
\frac{\partial(R+r)}{\partial \xi}=0
$$

but for $\varphi=\pi$ it is also true that

$$
\frac{\partial^{2}(R+r)}{\partial \xi^{2}}=0
$$

because $\xi_{0}$ and $\eta$ are on the caustic. Hence the only point to be considered is the one for which $\varnothing=\pi$. Then the integral can be simplified to

$$
\begin{aligned}
u & =\frac{i k \eta_{a}}{\pi} \int_{0}^{\infty} \frac{e^{i k(k+r)}}{R r} d \xi \int_{-\infty}^{\infty} \exp \left[-2 i k \frac{\sqrt{\xi \xi_{0} \eta \eta_{a}}}{R} \phi^{2}\right] d \phi \\
& =e^{i \frac{\pi}{\zeta}} \eta_{a} \sqrt{\frac{k}{2 \pi}} \int_{0}^{\infty} \frac{e^{i k(k+r)}}{R \sqrt{r \sqrt{\xi \eta_{a} \xi_{0} \eta}}} d \xi .
\end{aligned}
$$


Near the point of stationary phase $\bar{\xi}$ one can expard $R+r$ in a Taylor series

$$
R+r=(\bar{R}+\bar{r})+\frac{(\xi-\bar{\xi})^{3}}{6} A^{3}+\cdots
$$

where

$$
\left.A^{3}=\frac{\partial^{3}(R+r)}{\partial \xi^{3}}\right]_{\xi=\bar{\xi}}
$$

so that we obtain

$$
\begin{aligned}
u & =\frac{e^{i \pi / 4} \eta_{a} \sqrt{\frac{k}{2 \pi}} e^{i k(\bar{R}+\bar{r})}}{\bar{R} \sqrt{\bar{r} \sqrt{\xi \eta_{a} \xi_{0} \eta}}} \int_{-\infty}^{\infty} \exp \left[i k \frac{\xi^{3} A^{3}}{b}\right] d \xi \\
& =\frac{e^{i \pi / 4} \eta_{a} \sigma^{1 / 3} \Gamma\left(\frac{1}{3}\right) e^{i k(\bar{R}+\bar{r}) k^{1 / 6}}}{A \bar{R} \sqrt{3 \bar{r} \sqrt{\xi \eta_{a} \xi_{0} \eta}}} .
\end{aligned}
$$

In the immediate neighborhood of the cusp of the caustic we can use the following relations to simplify the expression for u:

$$
\begin{aligned}
& \bar{r} \approx a+\eta_{a} \\
& \bar{r} \approx \eta_{a}+\eta_{a}^{2} / a \\
& \xi_{0} \approx \eta_{a}^{2} / a \\
& \eta \approx \frac{16 \bar{b}^{3} a}{\left(a+\eta_{a}\right)^{2} \eta_{a}} \\
& A^{3} \approx \frac{6 a}{\left(a+\eta_{a}\right)^{2} \bar{\xi}}
\end{aligned}
$$

Then one obtains for $u$ near the cusp and on the caustic 


$$
u=\frac{e^{1 \pi / 4} \Gamma\left(\frac{1}{3}\right) \exp \left[i k\left(\frac{a+\eta_{a}}{a}\right)^{2}\right](a k)^{1 / 6}}{2 \sqrt{3}\left(a+\eta_{a}\right)^{1 / 3} \bar{\xi}^{2 / 3}} .
$$

Hence it may be observed that $u$ increases with $k$ like $k^{1 / 6}$ and that $u$ has simgularity at the cusp, but yet the behavior is different from that which is observed when the cusp is approached from above or below along the axis.

To analyze the case of line source on the axds of a parabolic cylinder we proceed in a similar manner by starting with

$$
u=2 e^{-i \pi / 4} \sqrt{\frac{2 k \eta_{a}}{\pi}} \int_{-\infty}^{\infty} \bar{u} \frac{e^{i k r}}{\sqrt{r}} d \sqrt{\xi}
$$

where

$$
\bar{u}=-H_{0}^{(I)}(k R) \approx-\sqrt{\frac{2}{\pi k R}} e^{1\left(k R-\frac{\pi}{4}\right)}
$$

so that

$$
a=\frac{4}{\pi} \sqrt{\eta_{a}} \int_{-\infty}^{\infty} \frac{e^{i k(R+r)}}{\sqrt{R r}} d \sqrt{\xi} .
$$

When the source is neither on the axis nor on the caustic it follows that

$$
u=o\left(\frac{1}{\sqrt{k}}\right)
$$

If we restrict ourselves to the exis we get

$$
u=\frac{8}{\pi} \sqrt{\eta_{a}} \int_{0}^{\infty} \frac{e^{i k(R+r)}}{\sqrt{k r}} d \sqrt{\xi} .
$$

If choose $\xi_{0}>\frac{\eta_{a}^{2}}{a}$ we have a point of etationary phase so that

$$
u=2 i e^{i k(\bar{R}+\bar{r})} \sqrt{2}\left[\pi k\left(\frac{a \bar{r}}{\bar{R}^{2}}+\frac{\xi_{0} \bar{R}}{\bar{r}^{2}}\right)\left(\sqrt{a \xi_{0}}-\eta_{a}\right)\right]^{-1 / 2} .
$$


The behavior of $u$ with respect to $k$ on this part of the axts is in no way distinguished from the rest of the space. But at the cusp of the caustic u does have singularity.

For $\xi_{0}<\eta_{a}^{2}$ a there is no stationary point and, se before, we replace the variable of integration $\xi$ by $x / k$ and discard all teras which vanish rapidly with respect to $k$. Then

$$
u=21 e^{i k(\bar{R}+\bar{r})} \sqrt{\frac{2 \eta_{a}}{\pi k\left(\eta_{a}^{2}-a \xi_{0}\right)}} .
$$

Thus the behavior of $u$ on the axis above and below the cusp of the caustic is essentially the same, nor does it behave very differently in the rest of the space, except that at the cusp a singularity aimflar to a line source, in an asymptotic sense, seems to exist.

On the axis, in the irmediate neighborhood of the cusp, the function $R+r$ can be expanded into

$$
R+r=a+\beta \xi+r \xi^{2}
$$

where

$$
\begin{aligned}
& a=a+\eta_{a}^{2} / a+2 \eta_{a}, \\
& \beta=2 \frac{\eta_{a}^{2}-a \xi_{0}}{\left(\eta_{a}+a\right)\left(\eta_{a}+\xi_{0}\right)}, \\
& r=2 \eta_{a}\left[\frac{a}{\left(\eta_{a}+a\right)^{3}}+\frac{\xi_{0}}{\left(\eta_{a}+\xi_{0}\right)^{3}}\right],
\end{aligned}
$$

and $\xi_{0} \approx \eta_{a}^{2} / a_{0}$. Then

$$
\begin{aligned}
& u=\frac{\frac{4}{\pi} \sqrt{\eta_{a}}}{\left.\sqrt{\left(\eta_{a}+a\right)\left(\eta_{a}+\xi_{0}\right.}\right)} \int_{-\infty}^{\infty} \exp \left[1 k\left(\alpha+\beta \xi+\gamma \xi^{2}\right)\right] d \sqrt{\xi}, \\
& u \approx \frac{8 \sqrt{a} \exp \left[i k\left(\alpha-\frac{\beta^{2}}{\delta \gamma}\right)\right]}{\pi\left(\eta_{a}+a\right)(k \gamma)^{1 / 4}}\left\{c_{14}(\delta)-1 s_{14}(\delta)\right\},
\end{aligned}
$$


where $C_{14}(\delta)$ and $S_{14}(\delta)$ represent Hardy'o generalized Alry integral [5]. At the cusp the above reduces to

$$
u=\frac{\Gamma\left(\frac{1}{4}\right) e^{i \pi / 8}}{\pi}\left[\frac{8 a}{k\left(\eta_{a}+a\right)^{2}}\right]^{1 / 4} \exp \left[1 k\left(\sqrt{a}+\frac{\eta_{a}}{\sqrt{a}}\right)^{2}\right]
$$

which remains finite, but is on the order of $k^{-1 / 4}$ with respect to $k$.

A different behavior can be detected when the cusp is approached along the caustic. We will assume in the following that the point with the ccordinates $\left(\xi_{0}, \eta\right)$ Lies on the crustic. Then

$$
u=\frac{4}{\pi} \sqrt{\eta_{a}} \int_{-\infty}^{\infty} \frac{e^{i k(R+r)}}{\sqrt{R r}} d \sqrt{\xi}
$$

where

$$
\begin{aligned}
& \mathrm{R}=\sqrt{\left(\xi-\eta_{\mathrm{a}}-\mathbf{a}\right)^{2}+4 \xi \eta_{\mathrm{a}}}, \\
& \mathbf{r}=\sqrt{\left(\xi-\eta_{\mathrm{a}}-\xi_{0}+\eta\right)^{2}+4 \xi \eta_{\mathrm{a}}+4 \xi_{0} \eta-8 \sqrt{\xi \xi_{0} \eta_{\mathrm{a}}}} .
\end{aligned}
$$

The function $R+r$ hes two points at which it is stationary, but at only one of these do both the firat and second derivatives vanish. Therefore only this point will be considered. Then near that point we can write

$$
R+r=\bar{R}+\bar{r}+\frac{(\xi-\bar{\xi})^{3}}{6} A^{3}+\cdots,
$$

where

$$
\left.A^{3}=\frac{\partial^{3}(R+r)}{\partial \xi^{3}}\right] \xi=\xi
$$

so that 


$$
\begin{aligned}
u & =\frac{2}{\pi} \sqrt{\frac{\eta_{a}}{\bar{R} \bar{r} \bar{\xi}}} \int_{-\infty}^{-30-} \exp \left[1 k \frac{\xi^{3} A^{3}}{6}\right] d \xi \\
& =\frac{2 \Gamma\left(\frac{1}{3}\right)}{\pi} \sqrt{\frac{\eta_{a}}{3 \bar{R} \bar{r} \bar{\xi}}} \frac{6^{1 / 3}}{k^{1 / 3} A} .
\end{aligned}
$$

Near the cusp of the caustic this expression reduces to

$$
u=\frac{2 \Gamma\left(\frac{1}{3}\right)}{\pi}\left[\frac{a}{27 \bar{\xi} k^{2}\left(a+\eta_{a}\right)^{2}}\right]^{1 / 6} \text {. }
$$

It may be observed that for fixed $k$, $u$ increases as the cusp is approached along the axis. On the caustic $u$ behaves Like $\bar{\xi}^{-1 / 6}$, but on the axis $u$ behaves like $\xi^{-1 / 2}$. However at a fixed point not on the caustic u is of the order $k^{-1 / 2}$, but of the order $k^{-1 / 3}$ on the caustic away from the cusp, and of the order $k^{-1 / 4}$ at the cusp.

In conclusion it should be pointed out that in the preceding cases only examples of boundary-value problems were treated for which the incoming and outgoing waves cancelled on the surface. The cases where the normal derivatives cancel could be treated in a similar manner since the asymptotic forms of the Green's functions $G_{2}$ have been derived for both the parabolold of revolution as well as the parabolic cylinder. The resulting integrations reduce to the same evaluations by stationary phase techniques, so that in many instances the answers could almost be written at sight upon comparison with the equivalent boundary-value problem of the first kind.

\section{Acknowledgment}

The author wishes to express his appreciationto Frof. W. Magnus for suggesting this research and for his continued interest. 


\section{References}

[1] Buchholz, H. - Die konfluente hypergeometrische Funktion; Springer, 1953.

[2] Keller, J.B., - Asymptotic solutions of some diffraction problems; Lewis, R.M., and New York Univereity, Institute of Mathematical Sciences, Seckler, B.D. Mivision of Electromagnetic Research, Research Report No. EM-81, 1955.

[3] Kay, I. and - Asymptotic evaluation of the fleld at a caustic; J. of Keller, J.B. Appl. Phys. 25, 876-883, (1954). Also New York University, Institute of Mathematical Sciences, Division of Electromagnetic Research, Research Report No. EM-55, 1953.

[4] Hochstadt, H. - Addition theorems for the functions of the paraboloid of revolution; New York University, Institute of Mathematical Sciences, Division of Electromagnetic Research, Research Report No. BR-18, May, 1956.

[5] Watson, G.N. - A treatise on the theory of Bessel functions; Macmillan Co., New York, 1948, pp. $320 \mathrm{ff}$. 


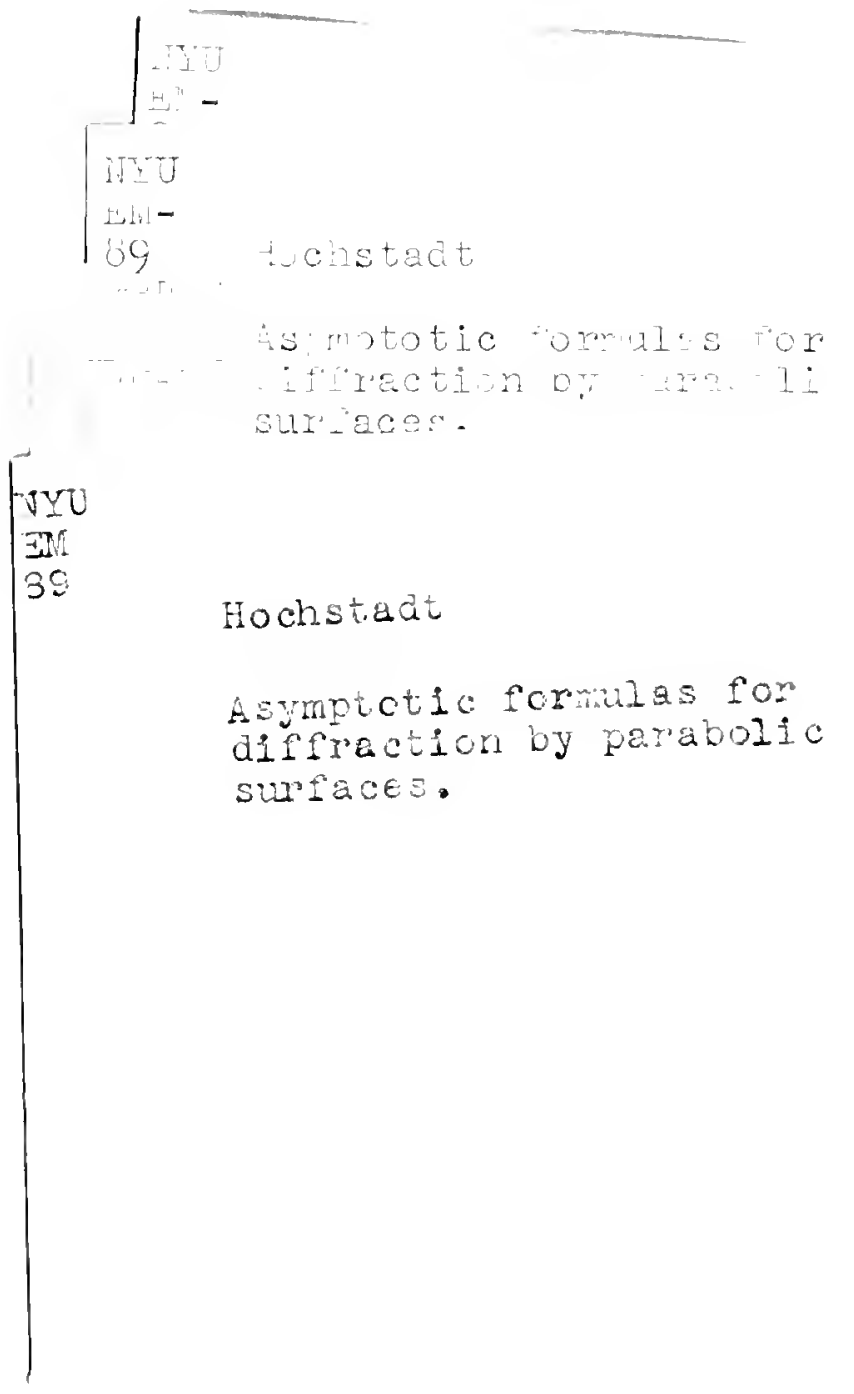


